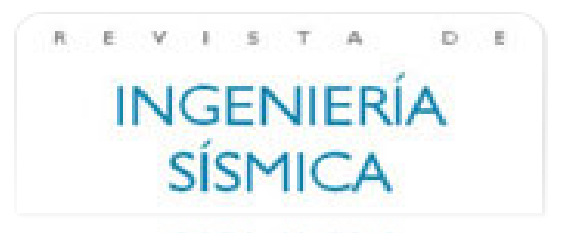

\section{Revista de Ingeniería Sísmica}

ISSN: 0185-092X

javiles@tlaloc.imta.mx

Sociedad Mexicana de Ingeniería Sísmica

México

Jaimes Téllez, Miguel Angel; Reinoso Angulo, Eduardo Comparación del comportamiento de edificios en el Valle de México ante sismos de subducción y de falla normal

Revista de Ingeniería Sísmica, núm. 75, 2006, pp. 1-22

Sociedad Mexicana de Ingeniería Sísmica

Distrito Federal, México

Disponible en: http://www.redalyc.org/articulo.oa?id=61807501

Cómo citar el artículo

- Número completo

- Más información del artículo

- Página de la revista en redalyc.org

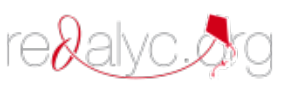

Sistema de Información Científica

Red de Revistas Científicas de América Latina, el Caribe, España y Portugal Proyecto académico sin fines de lucro, desarrollado bajo la iniciativa de acceso abierto 


\title{
COMPARACIÓN DEL COMPORTAMIENTO DE EDIFICIOS EN EL VALLE DE MÉXICO ANTE SISMOS DE SUBDUCCIÓN Y DE FALLA NORMAL
}

\author{
Miguel Ángel Jaimes Téllez y Eduardo Reinoso Angulo ${ }^{(1)}$
}

\begin{abstract}
RESUMEN
Se realiza un estudio comparativo del comportamiento que presentan algunas estructuras desplantadas en distintos sitios del valle de México, mediante la aplicación de un modelo simplificado (Miranda y Taghavi, 2005) ante eventos sísmicos simulados y registrados de subducción y falla normal. Se usa el método de simulación de funciones empíricas de Green (Ordaz y otros, 1995) y los sismos utilizados son los del 25 de abril de 1989 y del 21 de julio de 2000. Se analiza el comportamiento elástico estructural considerando dos tipos de deformación lateral: como viga de flexión y como viga de cortante, y se comparan los resultados en términos de los espectros de respuesta inelásticos, de la energía de entrada y de la energía histéretica normalizada de un sistema de un grado de libertad (1GDL). Los resultados muestran que un sismo de falla normal, debido al mayor contenido de alta frecuencia, provoca que los modos superiores de la estructura tengan mayor participación, lo que origina mayores intensidades en estructuras de periodo corto. Esto podría provocar mayores daños tanto en el edificio, como en sus contenidos con respecto a un sismo de tipo subducción. Para fines prácticos, se recomienda utilizar acelerogramas de diseño tanto de sismos de subducción como de falla normal y poder así evaluar mejor la respuesta estructural y el daño que ambos podrían provocar.
\end{abstract}

\begin{abstract}
A comparative study of the behavior of structures located at different sites in the valley of Mexico is shown using a simplified structural model (Miranda and Taghavi, 2005) and simulated strong ground motions for subduction and intermediate depth earthquakes. The strong ground motion simulation has been carried out using the empirical Green's functions method (Ordaz et al, 1995). The elastic behavior is analyzed considering two types of structural deformation: flexural beam and shear beam. The comparison is done using inelastic, input energy and normalized hysteretic energy spectra. Results show that the high frequency content of an intermediate depth earthquake yields a greater contribution from higher modes to the response and large intensities for short period structures. This could cause not only damage to the structure but also to its contents. From the earthquake resistant design point of view it is necessary to use design accelerograms from both subduction and intermediate depth earthquakes, in order to be able to evaluate the structural response and the damage that both types of strong ground motions could cause.
\end{abstract}

Artículo recibido el 16 de agosto de 2005 y aprobado para su publicación el 7 de marzo de 2006. Se aceptarán comentarios y/o discusiones hasta cinco meses después de su publicación.

(1) Instituto de Ingeniería, UNAM, Ciudad Universitaria, 04510 México, DF. migueljaimes@hotmail.com, ere@pumas.iingen.unam.mx 


\section{INTRODUCCIÓN}

Los sismos de subducción son los eventos sísmicos más estudiados en México por su frecuencia y magnitud y por la gran probabilidad de que pueda ocurrir en un futuro no lejano, un evento sísmico que podría superar lo ocurrido en 1985. Sin embargo, a lo largo de la historia se han presentado algunos eventos en el interior del país que liberan energía no por interacción entre las placas tectónicas como ocurre en los sismos de subducción sino asociados a un mecanismo de falla normal. Estos, en algunas ocasiones, han provocado no solamente daños estructurales y económicos, sino también pérdida de vidas humanas; ejemplo de ello son los sismos de Orizaba, Veracruz $(M w=7.0)$ en 1973, el de Huajuapan de León $(M w=7.0)$ en 1980 y más recientemente los del 23 de mayo de $1994(M w=6.5), 15$ de junio de $1999(M w=7.0)$ también en el mismo municipio de Huajuapan de León, 30 de septiembre de $1999(M w=7.5)$ en Oaxaca y 21 de julio de $2000(M w=5.9)$ en Puebla.

Existen evidencias históricas y avances recientes que muestran las diferencias entre estos dos tipos de sismos. Estudios detallados de la fuente indican que el tipo de falla, caída de esfuerzos y magnitud provocan que los sismos de falla normal sean más energéticos que los de subducción. Además, la propagación de las ondas de los sismos de subducción difiere de los sismos de falla normal (Furumura y Singh, 2002), donde la atenuación de los sismos de falla normal es mayor que la de los eventos de subducción (Singh, 2003). Como resultado de estas diferencias, el movimiento en la ciudad de México es muy distinto ante ambos eventos, reflejándose esto no sólo en el contenido de frecuencias donde los sismos de falla normal presentan un mayor contenido de alta frecuencia con respecto a los de subducción, sino también en la duración ya que los sismos de subducción son más largos que los de falla normal. Ante estas diferencias se puede esperar que los efectos que provocan los movimientos de subducción y falla normal en las estructuras sean diferentes.

Por otro lado, debido a la gran cantidad de factores que hay que tomar en cuenta en la evaluación de la respuesta de cualquier estructura en sus diferentes pisos es conveniente contar con herramientas prácticas para estimar las fuerzas impuestas por eventos sísmicos. Esto se debe a que en algunos casos no resulta práctico elaborar modelos detallados de una estructura para realizar una estimación rápida de las demandas de intensidad como la aceleración pico de piso, historias de tiempo de aceleración y espectros de piso para un movimiento sísmico dado. En este trabajo se utiliza el modelo simplificado de Miranda y Taghavi (2005) para analizar el comportamiento elástico de estructuras ya que se ha mostrado (Miranda y Taghavi, 2005; Taghavi y Miranda, 2005; Reinoso y Miranda, 2005) que el método da buenos resultados aun comparado con modelos muy elaborados de elementos finitos.

Para este estudio, se seleccionan dos sitios de la Ciudad de México: Ciudad Universitaria, CU, y Secretaría de Comunicaciones y Transportes, SCT, ubicados en terreno firme y blando, respectivamente y se consideran las dos fuentes sísmicas que más afectan a estos sitios (Guerrero y Profundidad Intermedia Centro donde se originan eventos de tipo subducción y falla normal, respectivamente). Mediante la aplicación de un método que considera la desagregación del peligro sísmico (Ishikawa y Kameda, 1988) es posible obtener los valores esperados de magnitud, 
$M$, y distancia, $R$, para generar una intensidad sísmica como la aceleración máxima del suelo o la aceleración espectral (en este trabajo, la aceleración máxima del suelo, $A s_{M A X}$ ) asociada a un periodo de retorno, TR, de las fuentes sísmicas estudiadas (Jaimes y otros, 2004). Esta técnica permite identificar los escenarios en términos de magnitud y distancia de la fuente al sitio que tiene una contribución significativa en el peligro sísmico para una tasa de excedencia dada. Se aplica con la finalidad de mostrar las diferencias de comportamiento que presentaría la estructura ante ambos eventos teniendo estos la misma intensidad sísmica en el sitio de interés.

El objetivo de este trabajo, es estudiar las diferencias de comportamiento de estructuras desplantadas en el valle de México ante eventos sísmicos de subducción y falla normal. Estas diferencias, se estudian mediante la aplicación de un método simplificado para estudiar el comportamiento elástico y la amplificación que tiene la estructura en distintos pisos con respecto a la aceleración máxima del suelo considerando ambos tipos de deformación lateral: como viga de flexión y como viga de cortante. Asimismo, para sistemas de un grado de libertad (1GDL) se comparan los espectros de respuesta inelásticos, de energía de entrada y de energía de histéresis. Por último, la respuesta elástica ante eventos de tipo subducción y de falla normal se compara con tres edificios instrumentados en la Ciudad de México: dos en zona de lago y uno en terreno firme. Estos han estado sometidos a eventos sísmicos moderados que no han causado daños a las estructuras, es decir, que las estructuras mantienen un comportamiento elástico, por lo que es posible aplicar el método propuesto.

\section{FUENTES SÍSMICAS, SITIOS Y ACELEROGRAMAS SIMULADOS}

En la figura 1 se muestran los límites y ubicación de las dos fuentes sísmicas idealizadas como polígonos (Nishenko y Singh, 1987) en las que se lleva a cabo un proceso de acumulación y liberación de energía independiente del que ocurre en otras fuentes sísmicas, incluidas en este estudio y que afectan a la Ciudad de México: Guerrero (sombreado oscuro) y Profundidad Intermedia Centro (sombreado tenue). Asimismo, se señala la ubicación de los dos epicentros que corresponden a los escenarios obtenidos (círculos) por medio de la técnica de desagregación del peligro sísmico $(D P S)$ que presentan mayor probabilidad de generar una aceleración máxima del suelo, $A s_{\text {MáX }}$, asociada a un periodo de retorno de 125 años. El periodo de retorno es el usado para calcular el espectro de diseño para el reglamento de construcciones de la Ciudad de México (Ordaz y otros, 2000). Por tanto, se asume que tal periodo de retorno da niveles de intensidad que proporcionarán un nivel aceptable de riesgo. Para CU, la aceleración resultante para el periodo de retorno mencionado es de $A s_{M A X}=0.72 \mathrm{~m} / \mathrm{s}^{2}$ y para SCT es de $A s_{M A X}=1.78 \mathrm{~m} / \mathrm{s}^{2}$. Mediante la aplicación de la $D P S$ se obtuvo para CU los valores esperados de $M=8.1$ y $R=256 \mathrm{~km}$ debido a la fuente de Guerrero y $M=7.7$ y $R=112 \mathrm{~km}$ debido a la fuente de Profundidad Intermedia Centro: para el sitio SCT se obtuvo una $M=7.9$ y $R=262 \mathrm{~km}$ debido a la fuente de Guerrero y $M=7.6$ y $R=123 \mathrm{~km}$ para la fuente de Profundidad Intermedia Centro. Para generar los acelerogramas simulados en CU y SCT para los escenarios sísmicos determinados en términos de los valores esperados de la magnitud y distancia antes mencionada, se seleccionaron los registros obtenidos de los eventos 25/abril/1989 (subducción) y 21/julio/00 (falla normal). En la figura 1 igualmente se muestra la ubicación de algunos eventos de subducción (cuadrados) y de falla normal (triángulos) ocurridos entre 1994 y 2000, y que fueron registrados en edificios instrumentados 
que se utilizarán más adelante en este estudio. La tabla 1 contiene los datos necesarios para realizar las simulaciones: ubicación, magnitud, momento sísmico y caída de esfuerzos.

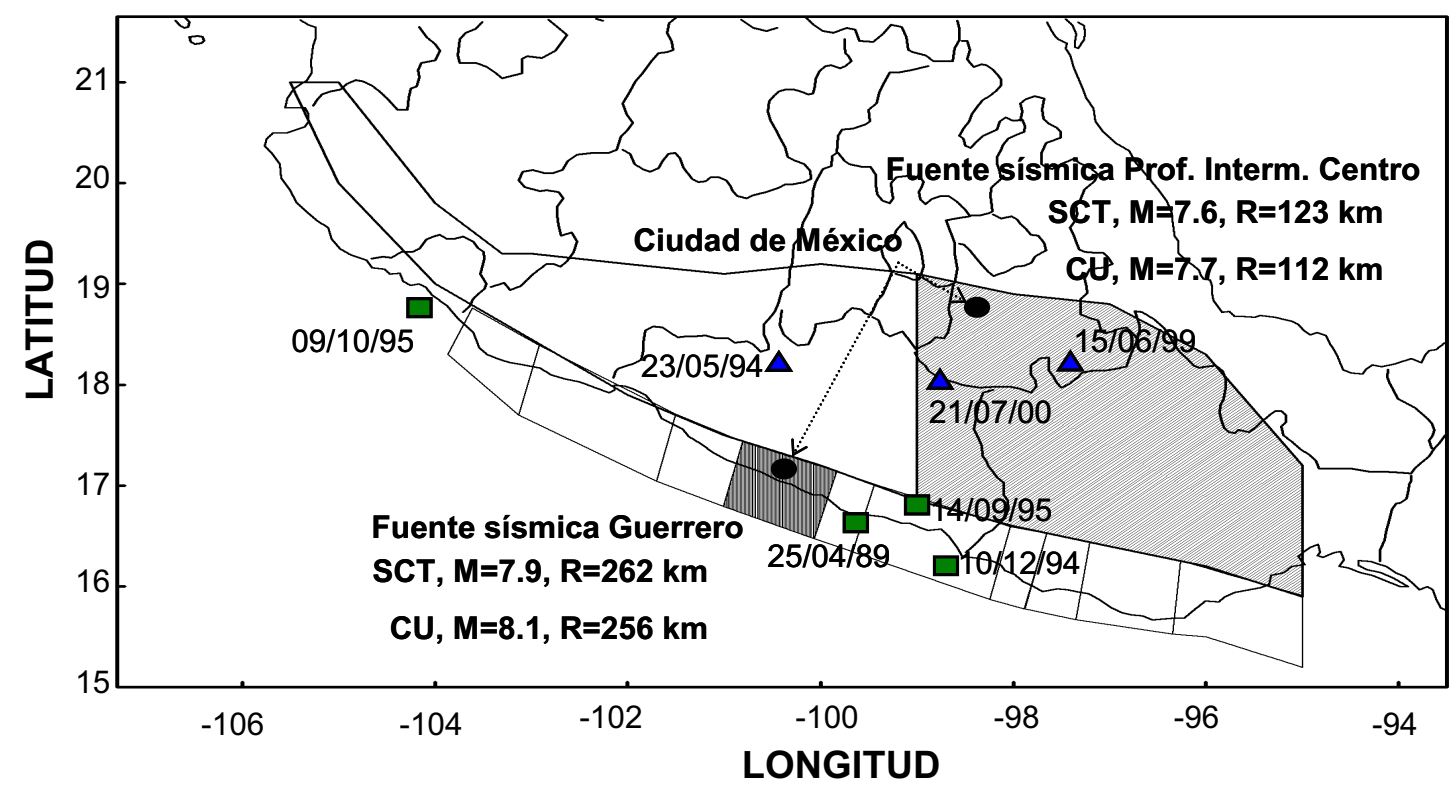

Figura 1. Localización de los eventos de subducción (rectángulos) y de falla normal (triángulos) utilizados en este estudio. También se indican los epicentros (círculos) y los valores esperados de M y R de las fuentes sísmicas de Guerrero (sombreado oscuro) y Profundidad Intermedia Centro (sombreado tenue) para generar eventos simulados en CU y SCT cuya aceleración máxima del suelo corresponde a un periodo de retorno de 125 años

Tabla 1. Datos de los sismos utilizados como funciones empíricas de Green

\begin{tabular}{|c|c|c|c|c|c|c|c|c|}
\hline & & & \multicolumn{2}{|c|}{ Epicentro } & & & & \\
\hline Evento & Sismo & $M_{s}$ & Lat & Long & Prof. & Tipo & $M_{o s}$ & $\Delta \sigma_{S}$ \\
\hline & & & $\left({ }^{\circ} \mathrm{S}\right)$ & $\left({ }^{\circ} \mathrm{W}\right)$ & $\mathrm{km}$ & & $($ dina-cm) & (bar) \\
\hline 1 & $25 / 04 / 89$ & 6.9 & 16.58 & 99.48 & 17 & SUB & ${ }^{1} 2.4 \times 10^{26}$ & ${ }^{1} 150$ \\
\hline 2 & $21 / 07 / 00$ & 5.9 & 18.09 & 98.97 & 16 & FN & ${ }^{2} 6.0 \times 10^{24}$ & ${ }^{2} 360$ \\
\hline
\end{tabular}

${ }^{1}$ Ordaz y otros (1995), ${ }^{2}$ Iglesias y otros (2002)

En la figura 2 se presentan los acelerogramas simulados obtenidos por medio del método de la función empírica de Green (FGE) con los valores esperados de magnitud y distancia para los sitios CU (lado izquierdo) y SCT (lado derecho) debido a las dos fuentes sísmicas estudiadas. En CU, se observa que para generar un sismo en la zona de Guerrero cuya $A s_{M A X}$ sea $0.72 \mathrm{~m} / \mathrm{s}^{2}$ el valor de la duración resultante es de $D \approx 80$ s y para zona de Profundidad Intermedia Centro de $D \approx 60$ s, es decir, que la duración en sismos de subducción resulta mayor que para los de falla normal; también se puede apreciar un mayor contenido de frecuencias bajas en el sismo de subducción con respecto al de falla normal. Para SCT (lado derecho) también resultan evidentes 
las diferencias de movimientos ante una misma intensidad, $A s_{M A X}=1.78 \mathrm{~m} / \mathrm{s}^{2}$, que generan las dos fuentes sísmicas en este sitio $(D \approx 160 \mathrm{~s}$, Guerrero y $D \approx 80 \mathrm{~s}$, Profundidad Intermedia Centro). Claramente, se ve que los efectos de estos dos grupos de simulaciones serían diferentes en cualquier estructura aun cuando tienen la misma intensidad en términos de la aceleración máxima del suelo, $A s_{M A X}$.

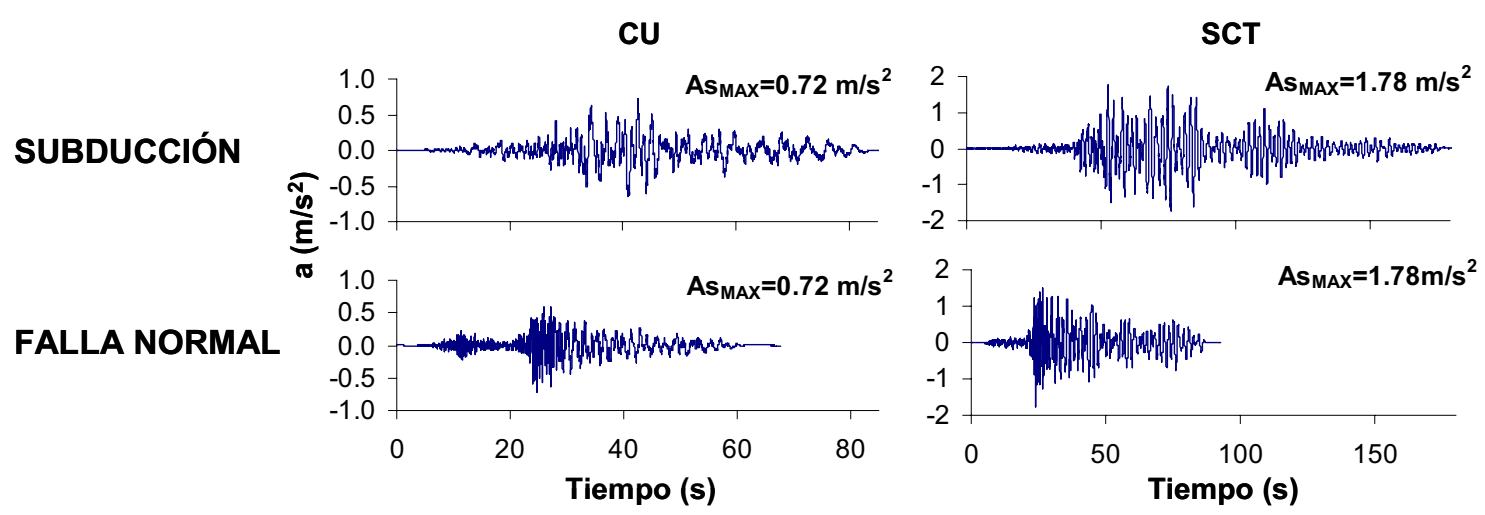

Figura 2. Acelerogramas simulados cuya aceleración máxima del suelo, $A s_{M A X}$, corresponde a un periodo de retorno de 125 años debidos a sismos de subducción (parte superior) y falla normal (parte inferior) en CU y SCT

\section{MODELO SIMPLIFICADO PARA EL CÁLCULO DE LA RESPUESTA ESTRUCTURAL}

Miranda y Taghavi (2005), desarrollaron un modelo para el cálculo de la respuesta estructural bajo comportamiento elástico lineal empleando un modelo continuo que consiste en una viga vertical en voladizo que combina deformaciones laterales de flexión y cortante cuando está sujeta a movimientos sísmicos horizontales en su base. El método combina la aplicación de un análisis modal utilizando, en nuestro caso, los seis primeros modos de vibrar con un modelo simplificado de la estructura a partir del cual se estiman formas modales, factores de participación y relación de frecuencias modales. El modelo está dado por la ecuación 1

$\frac{\rho}{E I_{o}} \frac{\partial^{2} u(x, t)}{\partial t^{2}}+\frac{1}{H^{4}} \frac{\partial^{4} u(x, t)}{\partial x^{4}}-\frac{\alpha^{2}}{H^{4}} \frac{\partial^{2} u(x, t)}{\partial x^{2}}=0$

donde $H$ es la altura total del edificio, $\rho$ la masa por unidad de longitud y $\alpha$ un parámetro adimensional. Este último parámetro está dado por la ecuación 2

$\alpha^{2}=H^{2} \frac{G A_{o}}{E I_{o}}$

donde $E I_{o}$ y $G A_{o}$ son la rigidez lateral en la viga de flexión y cortante, respectivamente. 
Si se considera que $\rho$ y la distribución de rigidez en altura son uniformes, las aceleraciones en cada nivel para una estructura con comportamiento elástico se pueden obtener como la superposición de la respuesta de todos los modos de vibrar según la ecuación 3

$$
\ddot{u}^{t}(x, t) \cong\left[1-\sum_{i=1}^{m} \Gamma_{i} \phi_{i}(x)\right] \ddot{u}_{g}(t)+\sum_{i=1}^{m} \Gamma_{i} \phi_{i}(x) A_{i}(t)
$$

donde $m$ es el número de modos considerados en la respuesta, $\Gamma_{i}$ el factor de participación del $i$ ésimo modo de vibrar, $\phi_{i}(x)$ la amplitud de la $i$-ésima forma modal a una altura $x, A_{i}(t)$ es la aceleración absoluta de un sistema de 1GDL con un periodo y amortiguamiento iguales a ese del $i$-ésimo modo de vibrar ante un movimiento del suelo $\ddot{u}_{g}(t)$.

En este trabajo se considerarán constantes en altura las propiedades dinámicas $\rho, G A_{o} \mathrm{y}$ $E I_{o}$ ya que Miranda y Taghavi (2005) observaron que en general la respuesta de edificios comunes no es sensible a la variación con la altura. El modelo del oscilador de varios grados de libertad requiere de tres parámetros: $T$, el periodo fundamental, $\xi$, el amortiguamiento crítico para las diferentes formas modales y $\alpha$. Éste último controla el grado de participación de las deformaciones laterales del oscilador causadas por un comportamiento de viga de cortante y viga de flexión, de manera que valores de $\alpha$ que tiendan a cero representan un oscilador que se deforma como viga de flexión, y valores de $\alpha$ grandes (mayores que 10) representan un oscilador que se deforma como viga de cortante. En el caso de edificios, aquellos cuya resistencia lateral está dada por muros de cortante tenderán a deformarse como una viga de flexión, y tendrán valores bajos de $\alpha$, y edificios formados por marcos tenderán a deformarse como una viga de cortante, y tendrán valores de $\alpha$ grandes (Miranda y Reyes, 2002).

\section{APLICACIÓN}

\section{Respuesta lineal de las estructuras analizadas}

Se presenta la respuesta de osciladores de varios grados de libertad ante los acelerogramas simulados en las estaciones CU y SCT, con periodos estructurales que van de 0.1 a 4 segundos. Se usa el método simplificado con $\alpha=4$ (que está dentro de los valores que corresponden a una deformación como viga de flexión) y $\alpha=10$ (que está dentro de los valores que corresponden a una deformación como viga de cortante), el amortiguamiento crítico considerado es del $5 \%$ para cada uno de los seis modos de vibrar. Esto se realiza para observar las diferencias de comportamiento de las estructuras ante los dos tipos de sismos, así como también denotar la importancia que toma el tipo de estructuración en la respuesta.

En la figura 3 se presentan las amplificaciones entre la aceleración máxima de cada piso del oscilador (PFA, Peak Floor Acceleration) y la aceleración máxima del suelo (PGA, Peak Ground Acceleration), PFA/PGA, para cuatro alturas relativas $(\mathrm{h} / \mathrm{H}=1.00,0.75,0.50$ y 0.25$)$ para 
osciladores de varios grados de libertad con periodos entre 0.1 a 4 segundos. La figura tiene resultados para CU (figura 3a) y para SCT (figura 3b) y los resultados son para los dos tipos de eventos. Las gráficas representan tres casos: 1) método simplificado con $\alpha=4$ (línea continua delgada), 2) $\alpha=10$ (línea continua gruesa) y 3) respuesta de un sistema de 1 GDL normalizado por la aceleración máxima del suelo (PGA) (línea discontinua). Para terreno firme (figura 3a) se observa que para $\mathrm{h} / \mathrm{H}<0.25$ la relación $\mathrm{PFA} / \mathrm{PGA}$ permanece casi constante ante la excitación de subducción, en cambio, para falla normal hay una mayor variación de ésta y depende sensiblemente para algunos periodos del tipo de estructuración dado por $\alpha$. La amplificación, PFA/PGA, en las diferentes alturas relativas, $\mathrm{h} / \mathrm{H}$, es mayor en periodos inferiores a un segundo para el sismo de falla normal que para el sismo de subducción; por otro lado, para el sismo de subducción se presentan amplificaciones más grandes en periodos mayores a un segundo. Asimismo, se puede apreciar que el espectro de respuesta del sistema de 1GDL normalizado por la aceleración máxima del suelo (línea discontinua) es muy similar al método simplificado para estructuras con periodos cortos, pero para periodos largos la amplificación en la azotea es menor. Esto se debe a que éste es un modelo de varios grados de libertad que considera distintas deformaciones laterales, y el sistema de 1GDL toma en cuenta sólo un modo y una deformación lineal. Sin embargo, el sistema de 1GDL da una buena idea de la forma como se amplifica el nivel superior con respecto al movimiento de la base. Además, se observa que el tipo de estructuración (dado por $\alpha$ ) es importante en los efectos de amplificación en los edificios para periodos largos, apreciándose más en los eventos de falla normal que en sismos de subducción. Esto se puede ver para estructuras con $\alpha=10$ cuya respuesta es distinta para periodos estructurales mayores a un segundo con respecto a estructuras con $\alpha=4$, donde los modos superiores tienen mayor participación. Es decir, que en marcos contraventeados o marcos con muros $(\alpha=4$, deformación a flexión) donde se esperan que las deformaciones a flexión tiendan a predominar sobre las deformaciones a cortante, para periodos estructurales largos las amplificaciones serán mayores con respecto a la aceleración máxima del suelo debido a sismos de falla normal. Por otro lado en terreno blando (figura $3 \mathrm{~b}$ ) se obtienen resultados similares a terreno firme. Se observa que para relaciones de $\mathrm{h} / \mathrm{H}<0.25$ las amplificaciones permanecen casi constantes; la amplificaciones son mayores para sismos de subducción con respecto a sismos de falla normal en periodos alrededor del periodo del suelo $\left(\mathrm{T}_{\mathrm{s}} \approx 1.9 \mathrm{~s}\right)$ y para sismos de falla normal en periodos menores a un segundo resulta una mayor amplificación, principalmente en el segundo modo de vibrar del suelo; el sistema de 1GDL predice las amplificaciones en la azotea para periodos menores a un segundo y los subestima en periodos más grandes ( $\mathrm{T}>1 \mathrm{~s})$. El tipo de estructuración (dado por $\alpha$ ) toma importancia para periodos largos principalmente ante sismos de falla normal pero en mucho menor medida que lo observado en terreno firme.

En la figura 4 se muestra la altura normalizada $(\mathrm{h} / \mathrm{H})$ contra las aceleraciones máximas de cada piso normalizadas a su vez con respecto a la del suelo (PFA/PGA) para diferentes osciladores caracterizados por su periodo. Los periodos mostrados son $\mathrm{T}=0.1,0.3,0.5,1,2,3$ y 4 segundos para $\mathrm{CU}$ y $\mathrm{T}=0.2,0.5,1,1.5,2,3$ y 4 segundos para $\mathrm{SCT}$, y corresponden a la respuesta de osciladores ante los sismos de subducción y falla normal con $\alpha=4$ (línea continua gruesa) y $\alpha=10$ (línea discontinua delgada). Nótese que la escala horizontal es distinta para todos los periodos. Para terreno firme (figura 4a) se advierte que para osciladores con periodo menor a un segundo los sismos de falla normal generan relaciones de amplificación, PFA/PGA, mayores 
Miguel Ángel Jaimes Téllez y Eduardo Reinoso Angulo

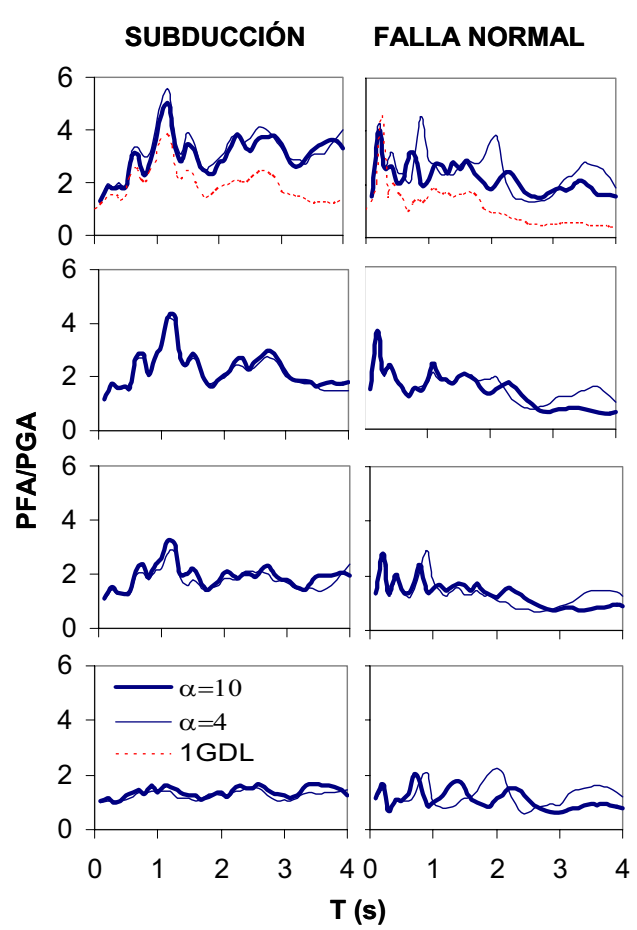

a) Estación CU en terreno firme

\section{SUBDUCCIÓN FALLA NORMAL}
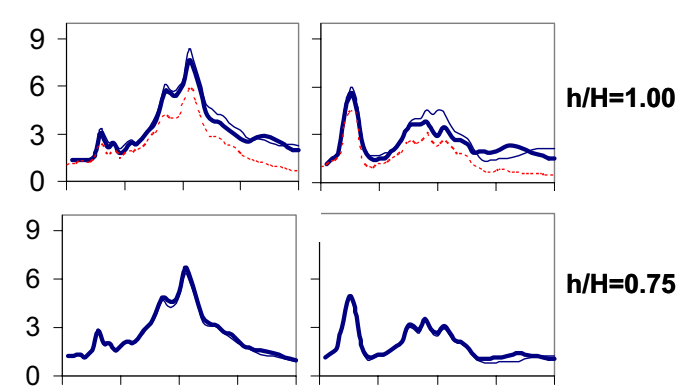

$\mathrm{h} / \mathrm{H}=\mathbf{0 . 7 5}$
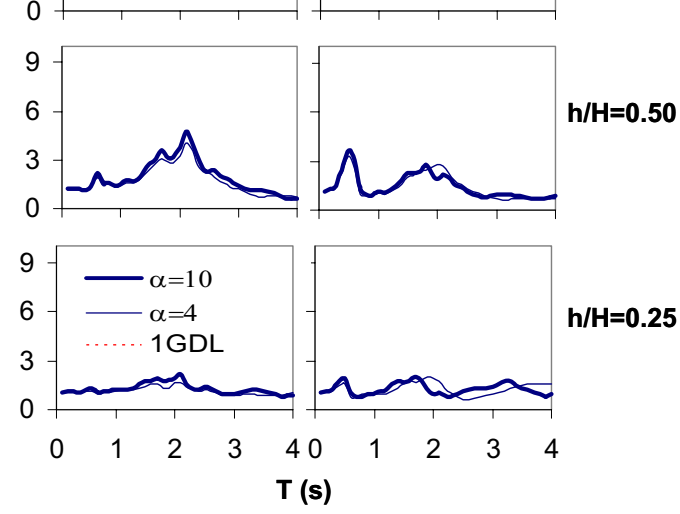

b) Estación SCT en terreno blando

Figura 3. Espectros de piso de aceleración normalizados con respecto a la aceleración del suelo (PFA/PGA) para cuatro alturas $(\mathrm{h})$ normalizadas $(\mathrm{h} / \mathrm{H})$ ante sismos de subducción y falla normal para tres condiciones: método simplificado con $\alpha=4$ y con $\alpha=10$, y respuesta de un oscilador de $1 \mathrm{GDL}$

que los sismos de subducción y para estructuras con periodo mayor a un segundo son los eventos de subducción los que provocan mayores amplificaciones en pisos superiores. Se aprecia que la relación PFA/PGA tiene una variación suave en las diferentes alturas relativas $(\mathrm{h} / \mathrm{H})$ en periodos menores a un segundo para ambos eventos debido principalmente a que el primer modo tiene mayor contribución en la respuesta estructural, es decir, que la participación de los modos superiores para este intervalo resulta de menor relevancia. Una excepción es el caso del oscilador con un periodo de $\mathrm{T}=0.3$ segundos sometido al sismo de falla normal donde existe una deamplificación con respecto a la base en $\mathrm{h} / \mathrm{H} \approx 0.25$ debida a la participación del segundo modo de vibrar. En cambio, los osciladores con periodo estructural mayor $(\mathrm{T}>1 \mathrm{~s})$, presentan una variación más irregular conforme el periodo crece; esto se debe porque los modos superiores comienzan a tener una mayor participación modal para ambos eventos. Pero es ante los eventos de falla normal donde se observa que los modos superiores tienen una mayor importancia (figura 4a parte inferior, $\mathrm{T}=1,2,3 \mathrm{y} 4 \mathrm{~s}$ ) debido al contenido de alta frecuencia en este tipo de sismos. Por otro lado, para estructuras con periodos estructurales menores a un segundo la configuración estructural (dado por $\alpha$ ) no varía significativamente, es decir, que la deformaciones laterales como viga de cortante o como viga de flexión (sistema de marcos, $\alpha=10$ y sistema de marcos con 

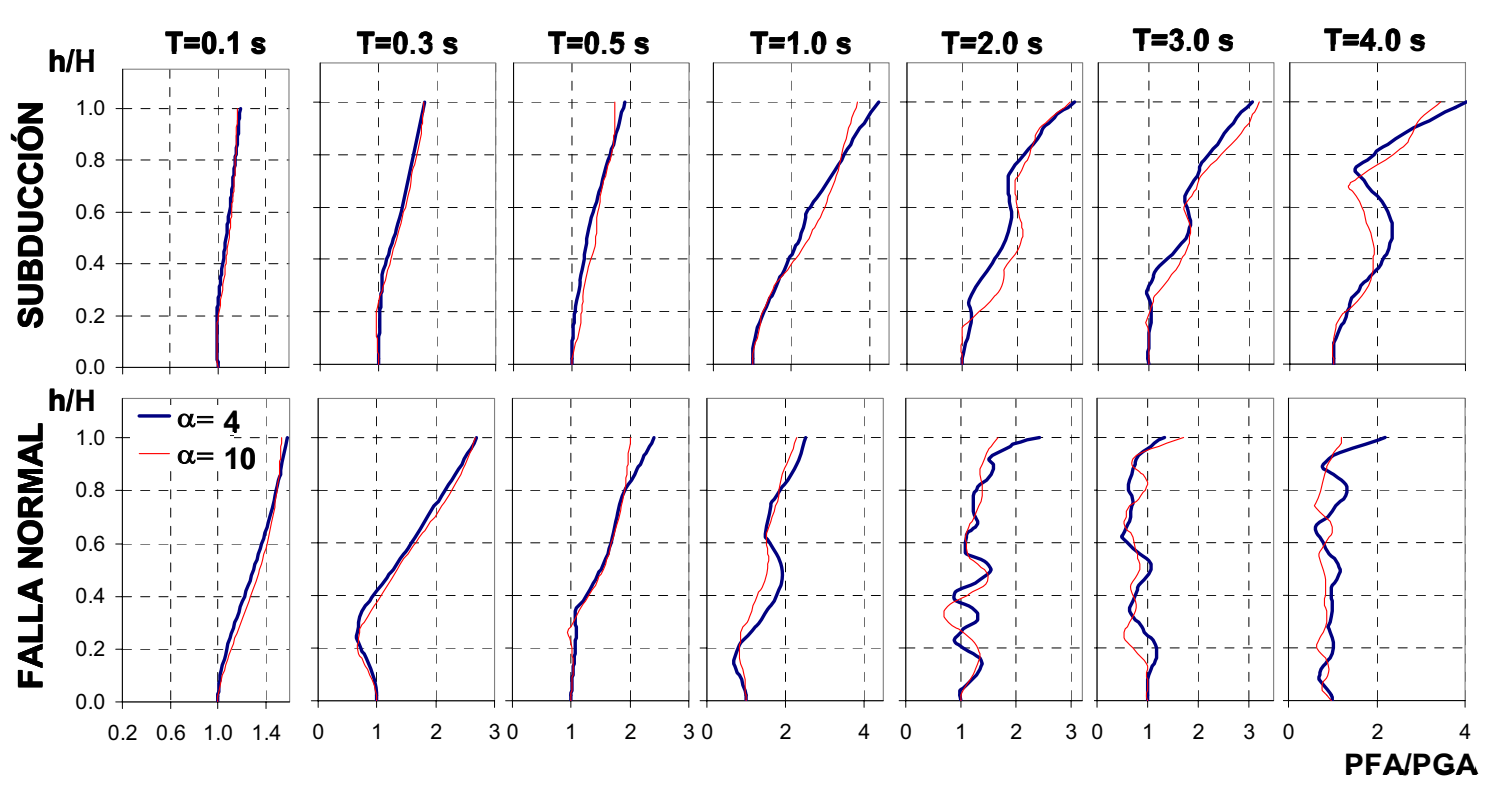

a) Estación CU en terreno firme
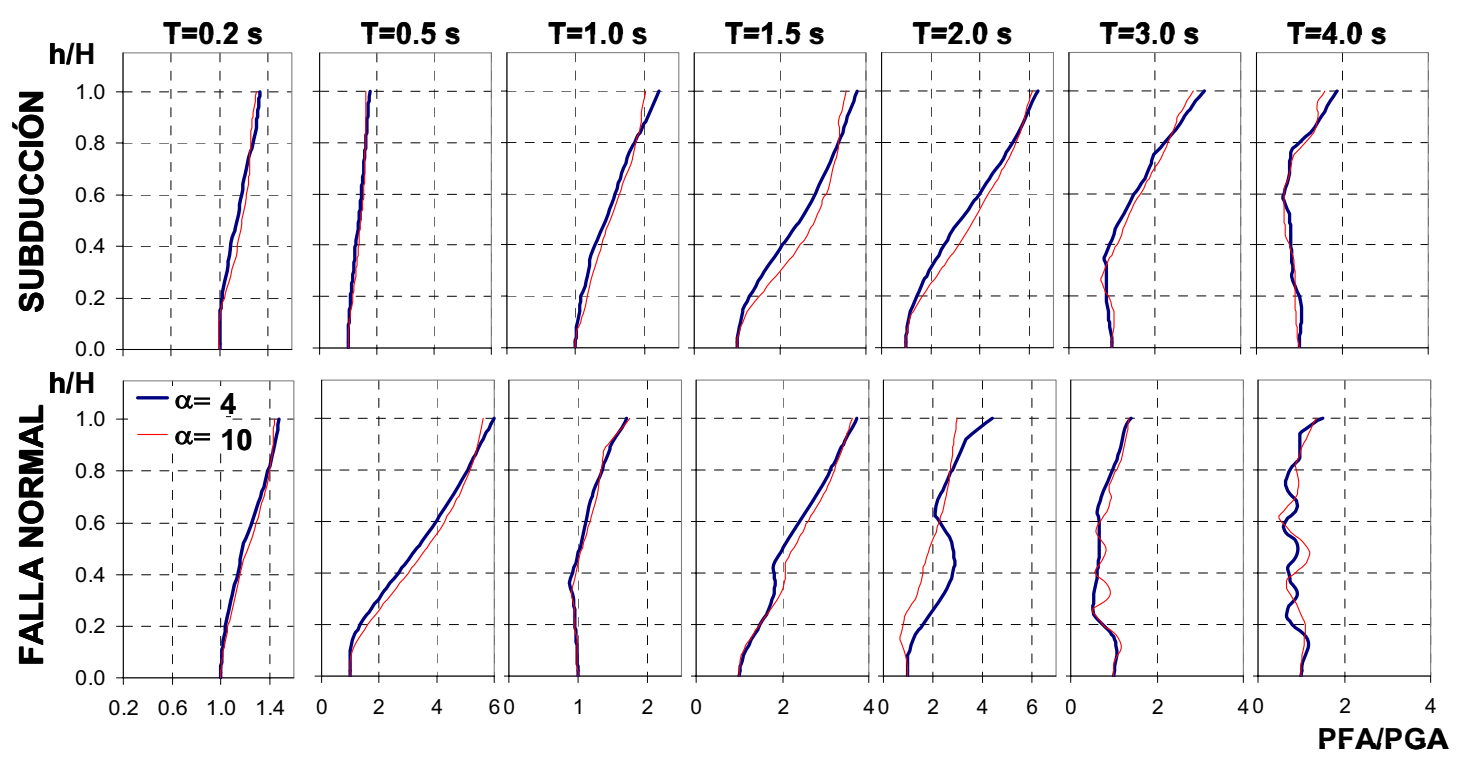

b) Estación SCT en terreno blando

Figura 4. Aceleración máxima de piso para distintos periodos estructurales normalizada con respecto a la del suelo (PFA/PGA) contra la altura normalizada $(\mathrm{h} / \mathrm{H})$ ante eventos de subducción y falla normal considerando dos tipos de deformación lateral: flexión $(\alpha=4)$ y cortante $(\alpha=10)$ 
muros de cortante o marcos contraventeados, $\alpha=4$ ) tanto en azotea como en pisos intermedios serán aproximadamente los mismos independientemente de la configuración tomada; en cambio, para estructuras con periodos mayores $(\mathrm{T}>1 \mathrm{~s})$ la configuración estructural influye en el tipo de movimiento lateral que tendrá la estructura tanto en azotea como en niveles inferiores, principalmente ante eventos de falla normal. Para terreno blando (figura 4b) se observan algunas similitudes a lo observado en terreno firme. La relación PFA/PGA es mucho mayor para el sismo de profundidad intermedia en periodos menores de 0.5 segundos con respecto al de subducción; la variación de PFA/PGA es suave en periodos menores al periodo del suelo $\left(\mathrm{T}_{\mathrm{s}} \approx 1.9 \mathrm{~s}\right)$ independientemente del tipo de evento sísmico y tiende a ser más irregular para periodos mayores, la configuración estructural toma mayor importancia en periodos mayores al periodo del suelo principalmente ante sismos de falla normal (figura $4 \mathrm{~b}, \mathrm{~T}=2,3 \mathrm{y} 4 \mathrm{~s}$ ).

\section{Espectros inelásticos}

En esta parte se analizan los espectros de respuesta elastoplásticos de un sistema de 1GDL en los sitios estudiados ante los dos tipos de fuentes sísmicas en las que se muestran diferencias importantes entre aquellos, tanto en terreno firme como en zona de lago, principalmente en periodos cortos. En la figura 5 se señala una representación espacial de los espectros inelásticos donde se observa la resistencia por unidad de masa $(\mathrm{R} / \mathrm{m})$ en cada periodo para que el sistema de 1GDL desarrolle la demanda de ductilidad requerida. La figura tiene resultados para CU (figura 5a) y SCT (figura 5b) y los resultados son para demandas de ductilidad de 1 a 4 . En estos cálculos se considera un modelo de un grado de libertad con comportamiento elastoplástico perfecto y un amortiguamiento de $5 \%$. Se observa para el sitio en terreno firme (figura 5a) que para periodos menores a un segundo la resistencia estructural que se debe proporcionar al sistema debido a sismos de falla normal es mayor para todos los valores de ductilidad que los valores de subducción; para periodos mayores a éste la resistencia está regida por eventos de tipo subducción. Para terreno blando (figura 5b) se puede apreciar algo similar donde la resistencia que se debe proporcionar a los sistemas con periodos estructurales entre 0.1 y 1 es mayor para los sismos de falla normal (principalmente para el segundo modo del suelo $\mathrm{Ts}_{2} \approx 0.5 \mathrm{~s}$ ), y para periodos mayores a estos (principalmente alrededor del periodo fundamental del suelo, $\mathrm{T}_{\mathrm{s}} \approx 1.9 \mathrm{~s}$ ) son más importantes las demandas de resistencia debido a los de subducción.

\section{Energía de entrada}

La energía de entrada permite representar el daño acumulado del movimiento sísmico por considerar efectos importantes como la duración y la carga repetida. La aceleración máxima del suelo o de la respuesta de un oscilador no siempre tiene una correlación directa con la duración del movimiento del suelo. Se ha mostrado que la duración puede tener un efecto significante en las demandas de deformación inelástica y de disipación de energía (Mahin, 1980). Asumiendo que la duración es importante en el daño acumulado de la estructura se estudia el espectro de energía de entrada elástico $\left(E_{I}\right)$. La $E_{I}$ permite representar el daño potencial total del movimiento del terreno provocado por el sismo debido a que considera tanto la amplitud como la duración del evento. 

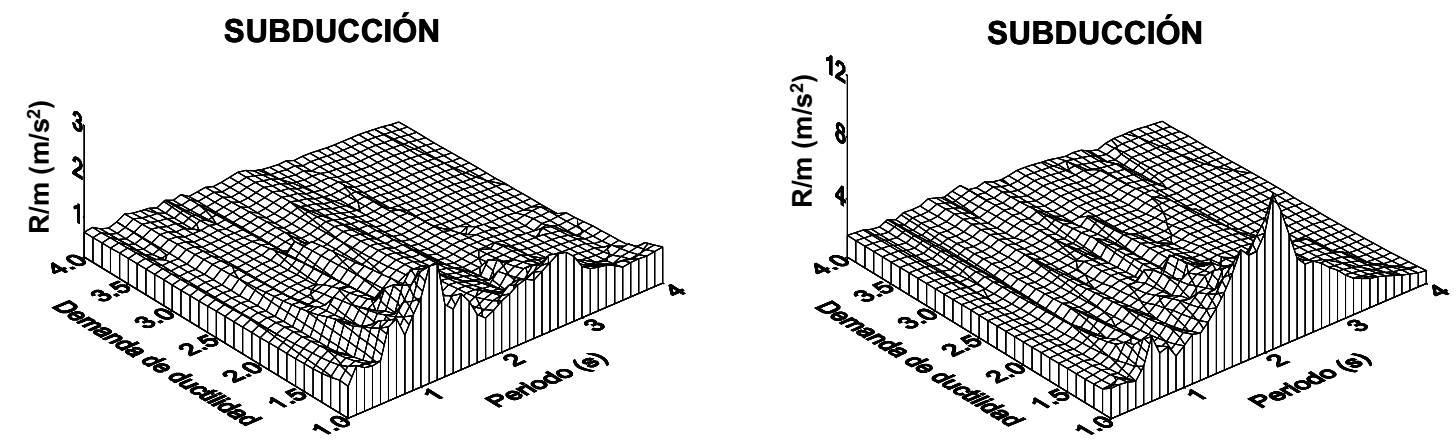

FALLA NORMAL

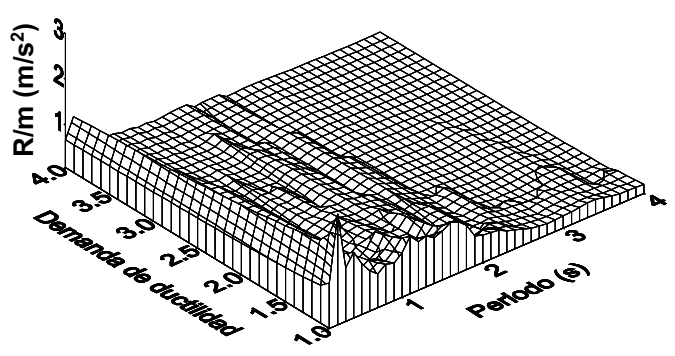

a) Estación CU en terreno firme

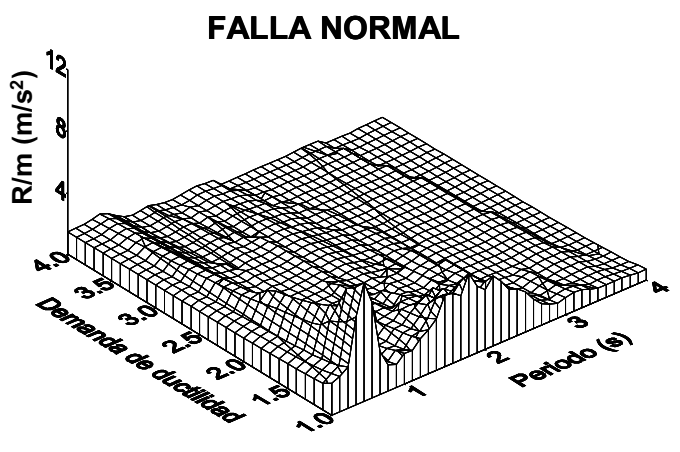

b) Estación SCT en terreno blando

Figura 5. Representación espacial de la variación de la resistencia por unidad de masa $(\mathrm{R} / \mathrm{m})$ con la demanda de ductilidad ante sismos de subducción (parte superior) y de falla normal (parte inferior)

En la figura 6 se aprecia la variación de los espectros de energía de entrada de los eventos simulados. Se observa que la $E_{I}$ es mucho mayor para el evento simulado de la brecha de Guerrero que para el de Profundidad Intermedia Centro (recuérdese que ambos movimientos tienen la misma $A s_{M A X}$ ). Esta diferencia es causada por una mayor duración y amplitud como se ve en la figura 2. Como era de esperarse la $E_{I}$ en terreno firme se concentra en periodos intermedios $(1<\mathrm{T}<2 \mathrm{~s})$ para el sismo de falla normal y en periodos relativamente largos para el de subducción (figura 6a). Se puede observar que los picos de los espectros de energía para CU están concentrados en periodos alrededor de $\mathrm{T} \approx 2.5$ y 1.5 segundos para subducción y falla normal, respectivamente. Esto es usual para la Ciudad de México, pero inusual para muchos sitios de terreno firme en el mundo, debido a que los sismos que afectan a dicha ciudad, se presentan a una mayor distancia con respecto a los que ocurren en otras partes del mundo, predominado el contenido de energía en periodos largos. Para SCT (figura 6b) se estima que la mayor concentración de energía está entre los periodos 1 y 3 , siendo mucho mayor la amplitud en el periodo fundamental del suelo $\left(\mathrm{T}_{\mathrm{s}} \approx 1.9 \mathrm{~s}\right)$ para las simulaciones de subducción. Para los eventos de profundidad intermedia la $E_{I}$ calculada presenta un pequeñísimo pico de $E_{I}$ en $\mathrm{T} \approx 0.5$ segundos. Esta concentración de energía es importante para estructuras con periodos similares a éste como se hace notar en la siguiente sección. 
SUBDUCCIÓN

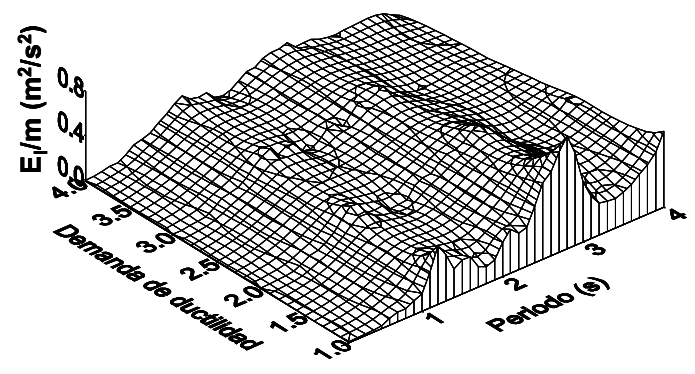

FALLA NORMAL

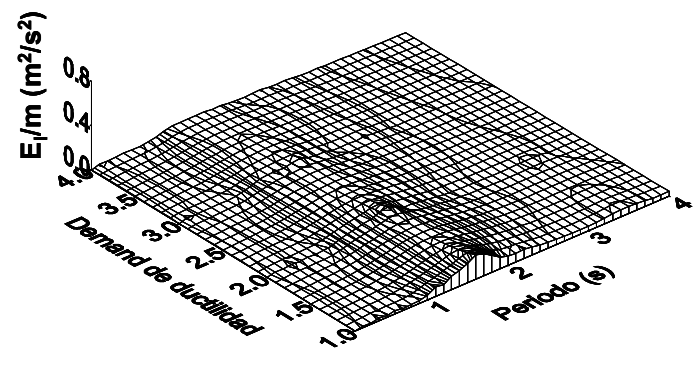

a) Estación CU en terreno firme
SUBDUCCIÓN

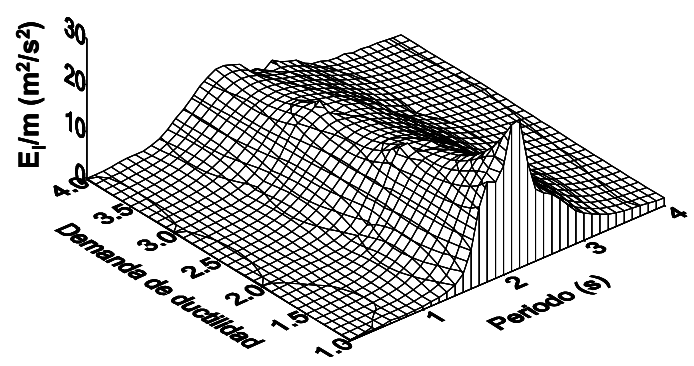

FALLA NORMAL

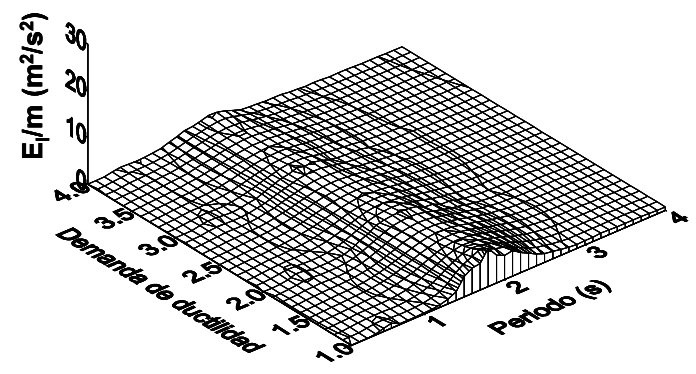

b) Estación SCT en terreno blando

Figura 6. Representación espacial de la variación de los espectros de energía de entrada para sismos de subducción (parte superior) y de falla normal (parte inferior)

\section{Energía de histéresis normalizada}

Un parámetro que se correlaciona bien con las demandas acumuladas de deformación plástica y por tanto, con el daño que la estructura pueda tener es la energía de histéresis normalizada, $N E_{H}$ (Terán-Gilmore, 1996) dada por la ecuación 4

$$
N E_{H}=\frac{E_{H}}{x_{y} F_{y}}
$$

donde $E_{H}$ es la energía de histéresis, $x_{y}$ el desplazamiento de fluencia y $F_{y}$ la resistencia de fluencia. El producto de estos dos últimos valores representa una proporción de la energía elástica de deformación almacenada cuando los elementos estructurales son llevados hasta el nivel de fluencia. Terán-Gilmore (1996) indica que valores similares de $E_{H}$ pueden estar asociados a sistemas que tienen gran resistencia y pocos ciclos de carga y a sistemas que tienen poca resistencia y muchos ciclos de carga. Para diferenciar la severidad de las deformaciones plásticas acumuladas en casos como estos puede utilizarse el concepto de $N E_{H}$. 
En la figura 7 se muestra la representación espacial de los espectros de $N E_{H}$ de los eventos simulados para distintas demandas de ductilidad. Se observa (puntos negros) para terreno firme (figura 7a) que para periodos $\mathrm{T}<2$ segundos la $N E_{H}$ resulta mayor para los de profundidad intermedia $\left(N E_{H} \approx 40\right)$ con respecto a los de subducción $\left(N E_{H} \approx 20\right)$. Para $\mathrm{CU}$, al igual que lo mostrado en la figura $6 \mathrm{a}$, los movimientos demuestran un alto contenido de energía para valores relativamente largos de periodo. Para SCT (figura $7 \mathrm{~b}$ ) se observa una pequeña diferencia en periodos menores a un segundo para subducción $\left(N E_{H} \approx 20\right)$ y para falla normal $\left(N E_{H} \approx 40\right)$. Estos resultados indican que los eventos de subducción son sin duda determinantes en el riesgo sísmico de estructuras en estos sitios del valle de México; sin embargo, se puede esperar que los sismos de falla normal puedan causar daños en estructuras con periodos de $0<\mathrm{T}<1.0$ segundos en terreno firme y en menor medida en zona de lago para periodos cortos.

\section{SUBDUCCIÓN}

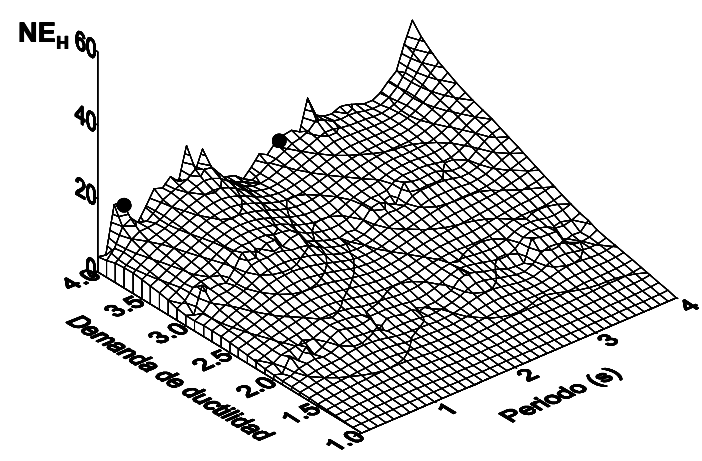

FALLA NORMAL

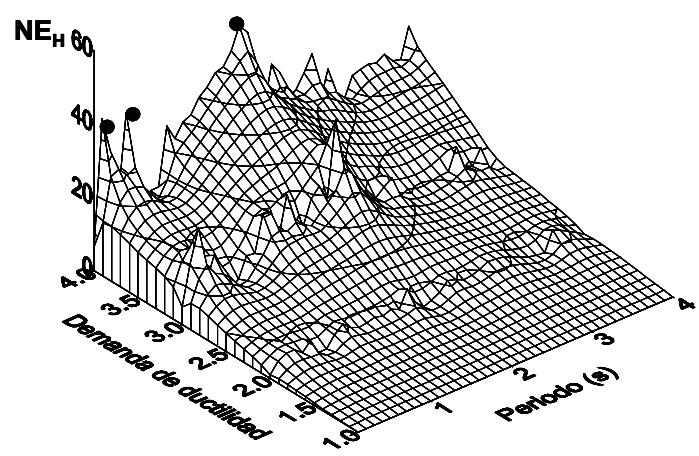

a) Estación CU en terreno firme

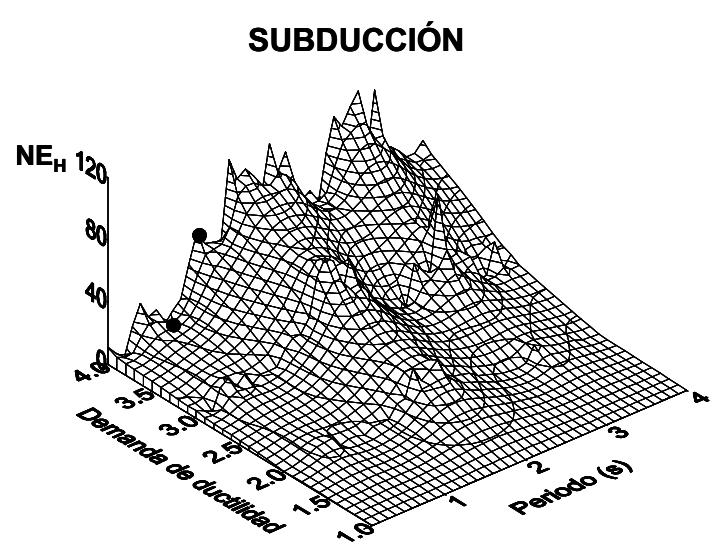

FALLA NORMAL

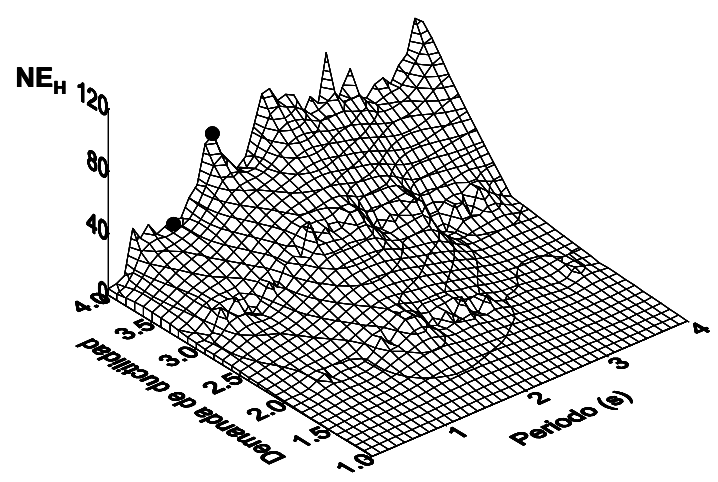

b) Estación SCT en terreno blando

Figura 7. Representación espacial de la variación de los espectros de energía de histéresis normalizada para sismos de subducción y de falla normal 


\section{OBSERVACIONES DE EDIFICIOS INSTRUMENTADOS ANTE EVENTOS REGISTRADOS DE SUBDUCCIÓN Y FALLA NORMAL}

Se estudiaron tres edificios instrumentados que tuvieran registrados en sus diferentes niveles eventos ocurridos en México entre 1994 y 2000 debido a sismos de subducción y falla normal (figura 1). Las respuestas observadas, se comparan con las calculadas por medio del método simplificado con la finalidad de observar la respuesta en otros niveles de los edificios donde no se tiene instrumento debido a que en algunos casos, sólo se cuenta con registros en la azotea y se desconoce cómo se comporta en pisos intermedios. Para ello, se determinan los parámetros requeridos como el periodo de la estructura, $T$, amortiguamiento, $\zeta$, y el parámetro $\alpha$. La tabla 2 contiene datos de los eventos y parámetros utilizados al emplear el método simplificado: edificio, fecha y tipo de evento, magnitud y distancia, dirección analizada, aceleración máxima del suelo (PGA, Peak Ground Acceleration), periodo de la estructura, amortiguamiento y el parámetro $\alpha$. Los parámetros se calculan para cada estructura y en cada dirección, es decir, que estos varían de evento a evento debido a que se ha observado que el periodo y amortiguamiento de algunas estructuras que han presentado comportamiento no lineal o han sido reestructuradas se han modificado en los últimos diez años (Arredondo y otros, 2004). Los edificios seleccionados son: PC y MJ en zona de lago y el edificio PI en terreno firme de la Ciudad de México.

Tabla 2. Parámetros empleados de eventos registrados en edificios instrumentados

\begin{tabular}{|c|c|c|c|c|c|c|c|c|c|}
\hline Edificio & Evento & Tipo & M & $\mathrm{R}$ & Dirección & $\begin{array}{l}\text { PGA } \\
\left(\mathrm{m} / \mathrm{s}^{2}\right)\end{array}$ & $\begin{array}{c}\mathrm{T} \\
(\mathrm{s}) \\
\end{array}$ & $\zeta$ & $\alpha$ \\
\hline \multirow{4}{*}{ PC } & \multirow{2}{*}{$10 / 12 / 94$} & \multirow{2}{*}{ SUB } & \multirow{2}{*}{6.6} & \multirow{2}{*}{290} & $\mathrm{~L}$ & 0.100 & 2.55 & 0.045 & 0.0 \\
\hline & & & & & $\mathrm{T}$ & 0.173 & 2.70 & 0.030 & 3.7 \\
\hline & \multirow{2}{*}{$21 / 07 / 00$} & \multirow{2}{*}{ FN } & \multirow{2}{*}{6.3} & \multirow{2}{*}{140} & $\mathrm{~L}$ & 0.110 & 2.72 & 0.029 & 0.0 \\
\hline & & & & & $\mathrm{T}$ & 0.130 & 2.72 & 0.020 & 4.5 \\
\hline \multirow{4}{*}{ MJ } & \multirow{2}{*}{$14 / 09 / 95$} & \multirow{2}{*}{ SUB } & \multirow{2}{*}{7.4} & \multirow{2}{*}{260} & $\mathrm{~L}$ & 0.320 & 0.65 & 0.048 & 3.5 \\
\hline & & & & & $\mathrm{T}$ & 0.320 & 0.68 & 0.050 & 4.0 \\
\hline & \multirow{2}{*}{$15 / 06 / 99$} & \multirow{2}{*}{ FN } & \multirow{2}{*}{6.5} & \multirow{2}{*}{230} & $\mathrm{~L}$ & 0.210 & 0.65 & 0.048 & 3.5 \\
\hline & & & & & $\mathrm{T}$ & 0.150 & 0.68 & 0.050 & 4.0 \\
\hline \multirow{4}{*}{ PI } & \multirow{2}{*}{$09 / 10 / 95$} & \multirow{2}{*}{ SUB } & \multirow{2}{*}{8.0} & \multirow{2}{*}{590} & $\mathrm{~L}$ & 0.02 & 1.50 & 0.009 & 3.1 \\
\hline & & & & & $\mathrm{T}$ & 0.02 & 1.49 & 0.014 & 8.0 \\
\hline & \multirow{2}{*}{$23 / 05 / 94$} & \multirow{2}{*}{ FN } & \multirow{2}{*}{6.2} & \multirow{2}{*}{210} & $\mathrm{~L}$ & 0.03 & 1.47 & 0.010 & 1.8 \\
\hline & & & & & $\mathrm{T}$ & 0.04 & 1.47 & 0.014 & 7.9 \\
\hline
\end{tabular}

\section{Edificio PC}

Es una estructura de 18 niveles formada originalmente por marcos de concreto reforzado en las dos direcciones; posteriormente, la dirección transversal y longitudinal fueron reforzadas con muros de concreto que se extienden desde la losa de sótano hasta la azotea (Pavón y Rodríguez, 1990). Se encuentra desplantado en terreno blando de la Ciudad de México, en un sitio con un periodo fundamental de 2.2 segundos. El edificio presenta un periodo estructural promedio de 2.63 y 2.71 en la dirección longitudinal y transversal respectivamente. En la tabla 2 se presentan 
los parámetros utilizados para obtener la respuesta mediante el método simplificado ante los dos eventos estudiados.

En la figura 8 se muestran las aceleraciones máximas observadas de cada piso normalizadas con respecto a la del suelo (PFA/PGA, puntos), y las calculadas con el método simplificado con respecto a su altura normalizada $(\mathrm{h} / \mathrm{H}$, línea continua), ante los sismos (ver figura 1) de subducción del 10 de diciembre de $1994(M w=6.6, R=290 \mathrm{~km})$ y de falla normal del 21 de julio de $2000(M w=6.3, R=140 \mathrm{~km})$. Se observa que a pesar de que las aceleraciones máximas del suelo son similares para ambos sismos (tabla 2), las amplificaciones, PFA/PGA, son mayores en los diferentes niveles para el evento de subducción (10/Dic/94) con respecto al de falla normal (21/Jul/00) por las razones expuestas en las figuras 3 y 4 . El modelo simplificado permite tener una mejor apreciación de estas diferencias y de los efectos que tienen estos dos tipos de eventos en la estructura. Estas diferencias son debidas a que los sismos de falla normal para estructuras con periodo estructural mayor al periodo del suelo (en este caso el periodo promedio de la estructura tanto en dirección longitudinal, $\mathrm{T} \approx 2.63 \mathrm{~s}$, como en dirección transversal, $T \approx 2.71 \mathrm{~s}$, resultan mayores al del periodo del suelo, $\mathrm{T}_{\mathrm{s}} \approx 2.2 \mathrm{~s}$ ) provocan que los modos superiores tengan mayor participación en la respuesta del edificio.

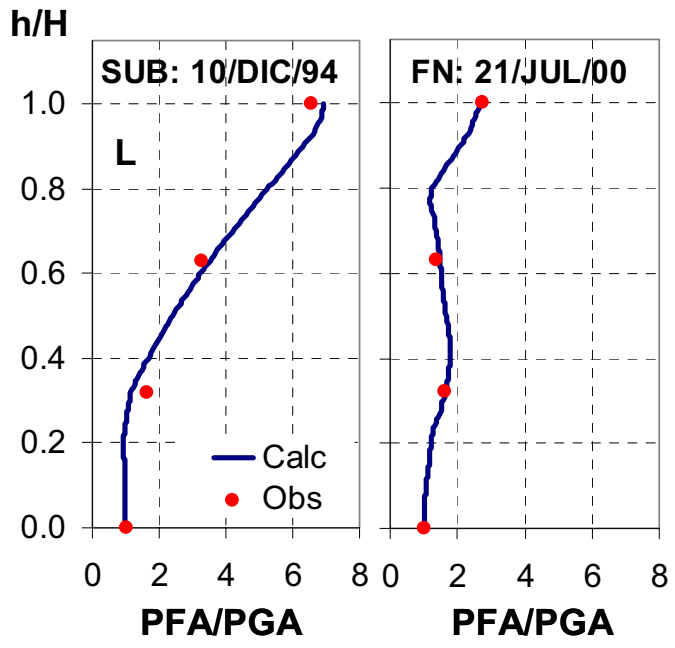

a) Dirección longitudinal

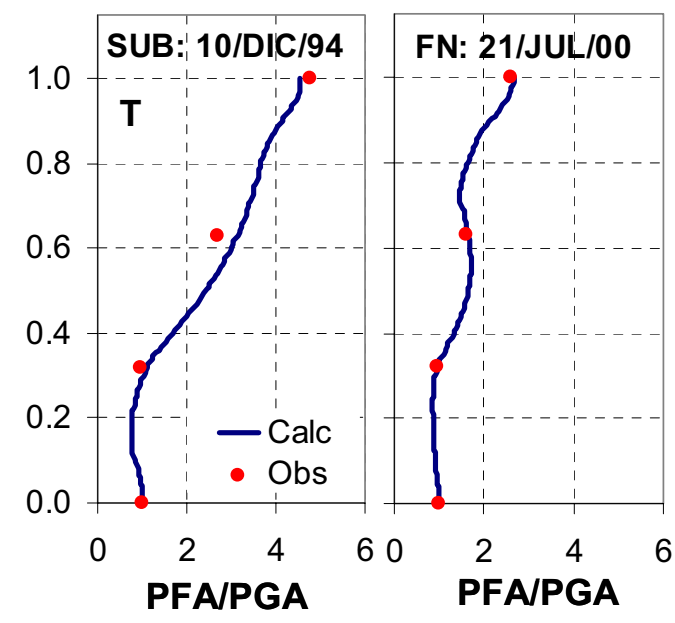

b) Dirección transversal

Figura 8. Aceleración máxima de piso normalizada con respecto a la del suelo (PFA/PGA) con respecto a la altura normalizada $(\mathrm{h} / \mathrm{H})$ ante un evento de subducción y de falla normal del edificio PC ubicado en zona de lago

Lo anterior también se aprecia en la figura 9 en la que se presentan los espectros de respuesta en diferentes niveles instrumentados $(\mathrm{h} / \mathrm{H}=1.00,0.63,0.32)$ y no instrumentados $(\mathrm{h} / \mathrm{H}=0.75$ y 0.15$)$ del edificio PC. Se observa cómo la respuesta observada es similar a la calculada con el método simplificado en los niveles instrumentados lo que valida el uso del método para extrapolar a pisos no instrumentados. Por otro lado, se ve que ante el evento de falla normal (21/07/00) la participación de los modos superiores (segundo y tercer modo principalmente) son mayores inclusive que la del primer modo de la estructura (excepto en el 
nivel $\mathrm{h} / \mathrm{H} \approx 0.75$ donde se presenta un punto de inflexión de los modos superiores a este nivel) en comparación con el sismo de subducción (10/12/94) donde el primer modo es dominante en ambas direcciones del edificio. Por tanto, la mayor participación de los modos superiores no sólo podría generar mayores distorsiones en pisos superiores sino además un mayor daño en los contenidos de los edificios debido a que son mayores las intensidades en los diferentes niveles en periodos menores con respecto al modo fundamental de la estructura o que, por ejemplo, un contenido con periodo de $0.8 \mathrm{~s}$ ubicado en cualquier piso (excepto nivel $\mathrm{h} / \mathrm{H} \approx 0.75$ ) sufra mayores demandas para un sismo de falla normal que para uno de subducción.

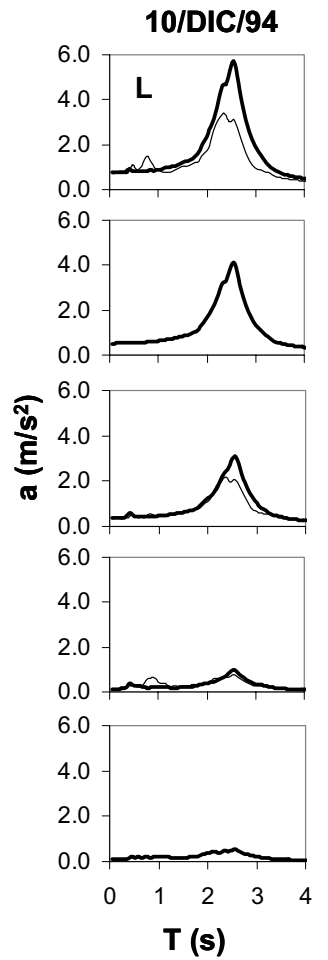

a) Dirección longitudinal

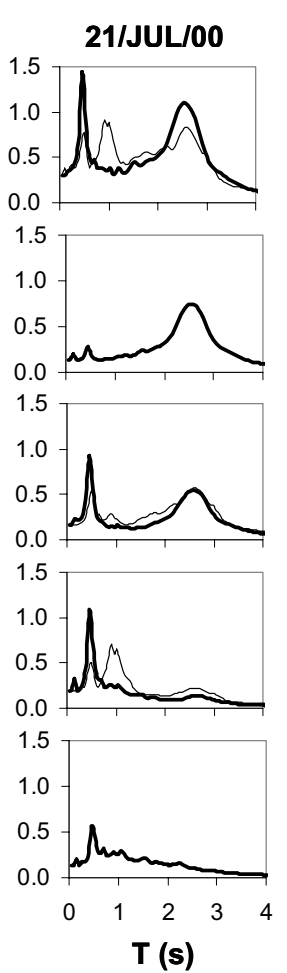

$\mathbf{T}(\mathbf{s})$

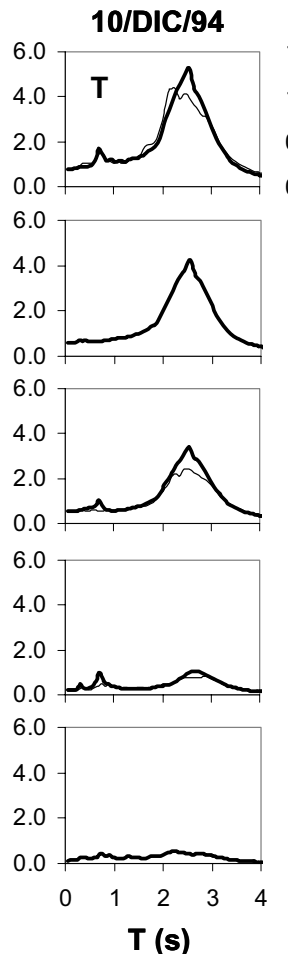

b) Dirección transversal

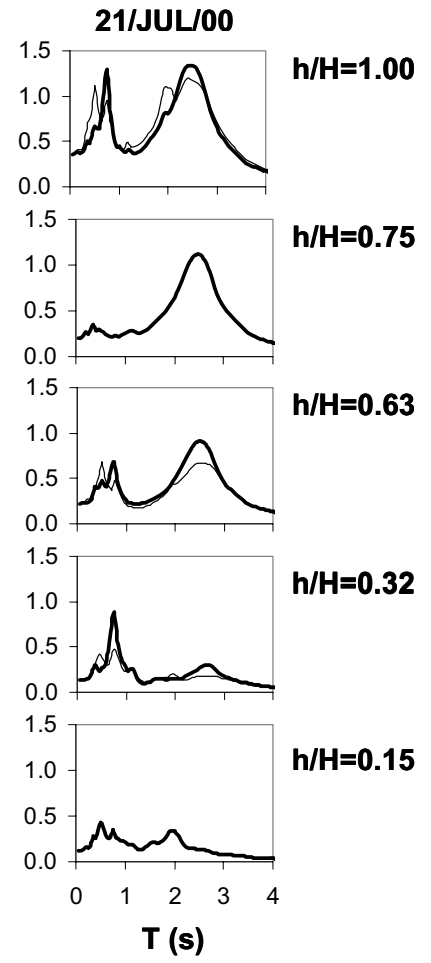

$\mathbf{T}(\mathbf{s})$

Figura 9. Respuesta en los diferentes niveles del edificio PC (línea delgada, observada y línea gruesa, calculada) ubicado en zona de lago ante los sismos del 10/Dic/94 y 21/Jul/00

\section{Edificio MJ}

Está ubicado cerca de la estación del metro Juárez. Tiene 7 niveles. La estructura original estaba formada por marcos de concreto reforzado y posteriormente fue reforzada con encamisado de columnas con placas de acero y colocando contraventeos de acero en ambas direcciones y muros de concreto en la dirección longitudinal. El edificio está desplantado en terreno blando de la Ciudad de México, en un sitio con un periodo fundamental de dos segundos. En la tabla 2 se indican los parámetros empleados al aplicar el método simplificado para reproducir adecuadamente la respuesta. La estructura tiene un periodo promedio de 0.65 y 0.68 en la 
dirección longitudinal y transversal, respectivamente, ante los dos eventos sísmicos analizados (ver figura 1) del 14 de septiembre de $1995(M w=7.4, R=260 \mathrm{~km})$ de subducción y el del 15 de junio de $1999(M w=6.5, R=230 \mathrm{~km})$ de falla normal. Estos dos eventos se consideraron en esta estructura porque se presentaron después de que el edificio fue reforzado en el año de 1992 con muros de concreto.

En la figura 10 se presentan al igual que en la 8 pero para el edificio MJ, las aceleraciones observadas de cada piso normalizadas con respecto a la del suelo (PFA/PGA, puntos), y las calculadas con el método simplificado con respecto a su altura normalizada $(\mathrm{h} / \mathrm{H}$, línea continua). En esta figura se puede observar que la respuesta obtenida por medio del método simplificado (línea continua) con respecto al observado (puntos) considerando sólo tres parámetros está bien aproximada, y que gracias al método simplificado se tiene una buena idea del tipo de configuración que presentó la estructura ante los dos eventos. La relación PFA/PGA es mayor para el evento de falla normal a pesar de que el sismo de subducción presenta una mayor aceleración del suelo $\left(\mathrm{PGA}=0.32 \mathrm{~m} / \mathrm{s}^{2}\right)$ que el de falla normal $\left(\mathrm{PGA}=0.21 \mathrm{~m} / \mathrm{s}^{2}\right)$; esto debido a que el contenido de alta frecuencia del sismo de falla normal provoca en este caso intensidades espectrales similares al sismo de subducción (ver figura 5). Asimismo, se observa lo que anteriormente se había mencionado (figura 4) que cuando el periodo de la estructura es menor que el periodo del suelo, el modo que más participa en la estructura debido a sismos de subducción y falla normal es el primer modo. Es decir, como el edificio tiene un periodo de $\mathrm{T} \approx 0.65 \mathrm{~s}$ en la dirección longitudinal y un periodo de $\mathrm{T} \approx 0.68 \mathrm{~s}$ en la dirección transversal (ambos menores que el periodo del suelo, $\mathrm{T}_{\mathrm{s}} \approx 2 \mathrm{~s}$ ) provoca que predomine el primer modo del edificio.

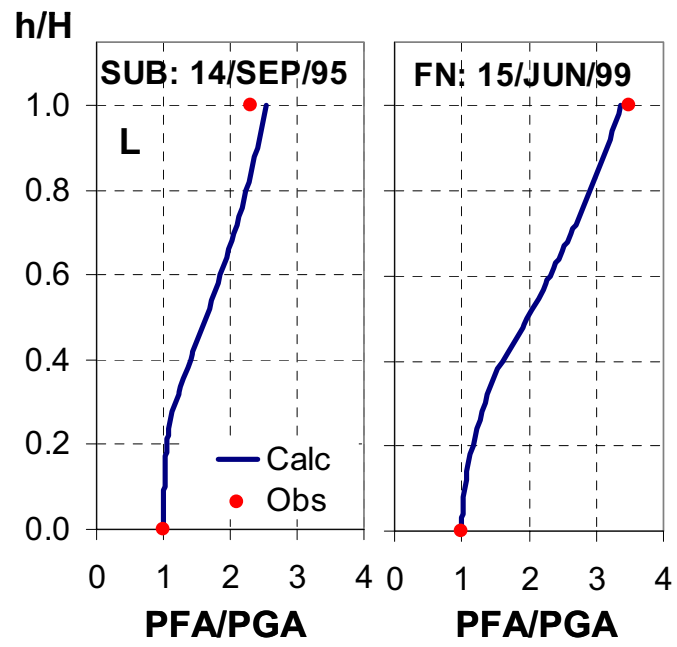

a) Dirección longitudinal

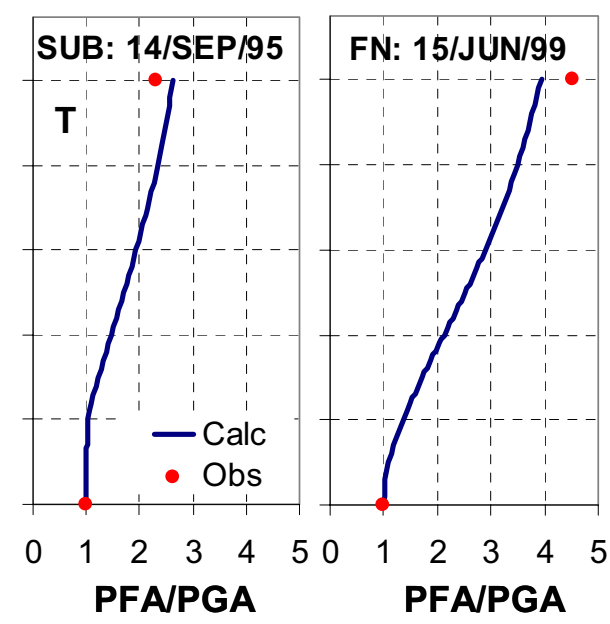

b) Dirección transversal

Figura 10. Aceleración máxima del piso normalizada con respecto a la del suelo (PFA/PGA) y con respecto a la altura normalizada $(\mathrm{h} / \mathrm{H})$ ante un evento de subducción y de falla normal del edificio MJ ubicado en zona de lago 
En la figura 11 se presentan los espectros de respuesta de piso de la azotea, único piso instrumentado en el edificio; se muestra cómo la respuesta obtenida con el método analítico (línea gruesa) es casi idéntica a la respuesta observada (línea delgada) y como es el primer modo $(\mathrm{T} \approx 0.65 \mathrm{~s}$ y de $\mathrm{T} \approx 0.68 \mathrm{~s}$ ) el que tiene mayor participación para ambos tipos de eventos.

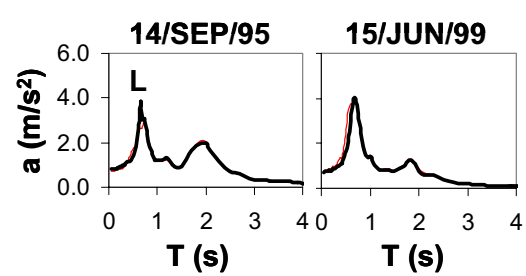

a) Dirección longitudinal

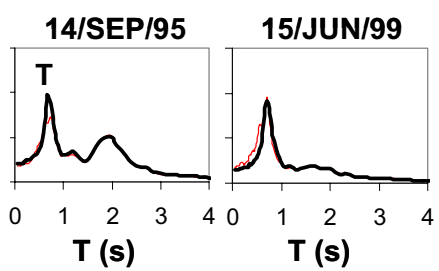

b) Dirección transversal

Figura 11. Respuesta en la azotea del edificio MJ (línea delgada, observada y línea gruesa, calculada) ubicado en zona de lago ante los sismos del 14/Sep/95 y 15/Jun/99

\section{Edificio PI (Torre IV)}

Esta estructura está integrada por cuatro torres (I, II, III y IV) de marcos de concreto reforzado con una altura mayor a $60 \mathrm{~m}$, unidas en $\operatorname{los} 6$ niveles inferiores. Las torres I y II fueron construidas en 1982 mientras que las III y IV en 1992. El edificio se encuentra instrumentado en las torres I y IV. Está desplantado en terreno firme de la Ciudad de México. Este edificio presenta un periodo estructural aproximado de 1.485 y 1.48 segundos en la torre IV y de 1.68 y 1.92 segundos en la torre I, en las direcciones longitudinal y transversal, respectivamente. Se analizó la torre IV porque se contaba con información sobre los dos tipos de sismos que se han manejado en el trabajo. En la tabla 2 se presentan los parámetros utilizados para reproducir por medio del método simplificado esta estructura ante los dos eventos analizados (figura 1): 9 de octubre de $1995(M w=8.0, R=590 \mathrm{~km})$ de subducción y el del 23 de mayo de $1994(M w=6.2, R=210 \mathrm{~km}) \mathrm{de}$ falla normal.

En la figura 12 se presenta al igual que en las figuras 8 (edificio PC) y 10 (edificio MJ) los cocientes PFA/PGA con respecto a su altura normalizada $(\mathrm{h} / \mathrm{H})$ tanto los observados (puntos) como los calculados (línea continua) para el edificio PI. Las observaciones hechas para los edificios desplantados en terreno blando son también válidas en terreno firme. La estructura ante el evento de falla normal presenta mayor participación de los modos superiores con respecto al de subducción. La mayor participación de los modos superiores en el sismo de falla normal (23/may/94) puede provocar mayores movimientos laterales en niveles superiores con respecto al observado de subducción (09/oct/94) como se indica en la figura con una línea horizontal para ambas direcciones del edificio. Se observa cómo la amplificación en pisos superiores es mayor ante el sismo de falla normal con respecto al de subducción.

En la figura 13 se presenta la respuesta tanto en niveles donde hay registro $(\mathrm{h} / \mathrm{H} \approx 1.00 \mathrm{y}$ $0.325)$ como donde no lo hay $(\mathrm{h} / \mathrm{H} \approx 0.75$ y 0.15$)$ del edifico PI (línea delgada, observada, y línea gruesa, calculada con el método simplificado). En esta figura se aprecia al igual que en el edificio PC (figura 9) cómo cuando la estructura tiene un periodo estructural mayor al periodo del suelo 
da como resultado que los modos superiores tengan mayor participación ante este tipo de eventos (excepto en el nivel $\mathrm{h} / \mathrm{H} \approx 0.75$ donde no hay contribución de modos superiores debido a que coincidentemente se presenta en esa altura un punto de inflexión). Esto implica, no sólo posibles daños en pisos superiores sino mayor daño en los contenidos del edificio.

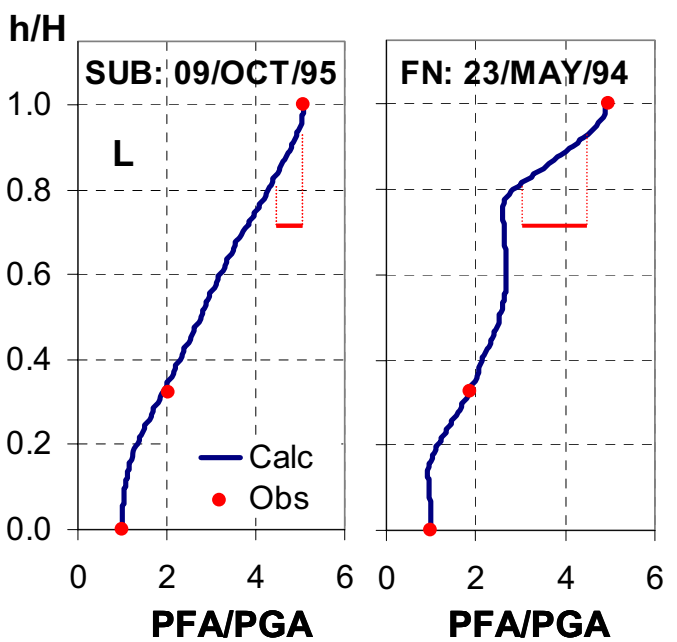

a) Dirección longitudinal

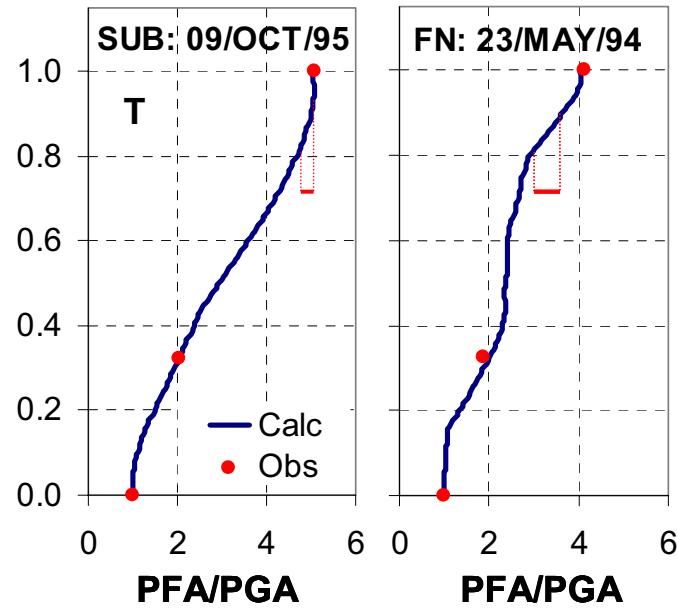

b) Dirección transversal

Figura 12. Aceleración máxima del piso normalizada con respecto a la del suelo (PFA/PGA) con respecto a la altura normalizada $(\mathrm{h} / \mathrm{H})$ ante un evento de subducción y de falla normal del edificio PI ubicado en terreno firme

\section{CONCLUSIONES}

En este trabajo, se presentaron las diferencias en la respuesta sísmica de estructuras ubicadas en el valle de México ante dos tipos de eventos: subducción y falla normal. Se muestran divergencias importantes en periodos cortos $(\mathrm{T}<1.0 \mathrm{~s})$ en los espectros de respuesta inelásticos, en la energía de entrada y en la energía histéresis normalizada de sistemas de 1GDL sobre todo en terreno firme, teniendo mayor intensidad los sismos de falla normal con respecto a los de subducción. Estos resultados indican que se pueden esperar mayores demandas inelásticas en las estructuras ante sismos de profundidad intermedia.

Se estudiaron eventos sísmicos registrados en edificios instrumentados tanto en terreno firme como en zona de lago de la Ciudad de México. Se encuentra que los modos superiores pueden tener una gran influencia cuando los sismos son de falla normal. Estos modos superiores provocan una mayor intensidad en periodos cortos que podrían afectar a los contenidos en las estructuras y causar daños en pisos superiores. 
Miguel Ángel Jaimes Téllez y Eduardo Reinoso Angulo

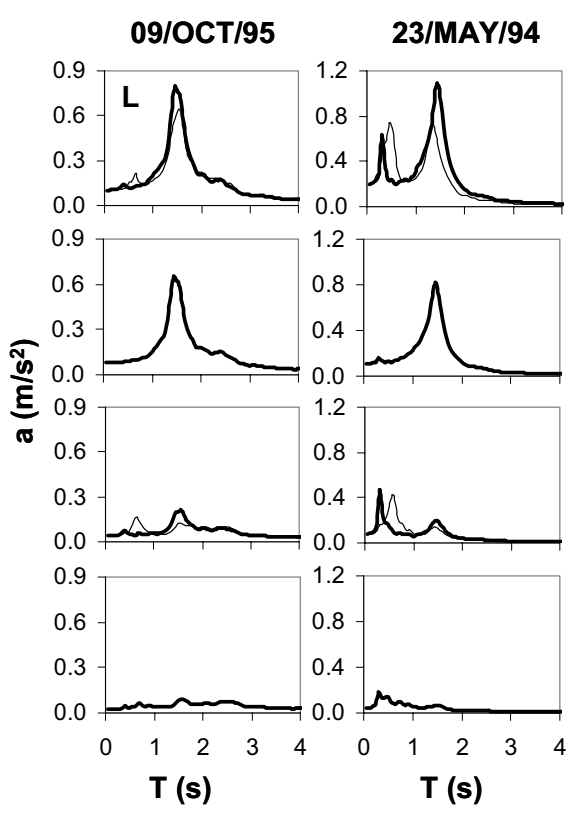

a) Dirección longitudinal

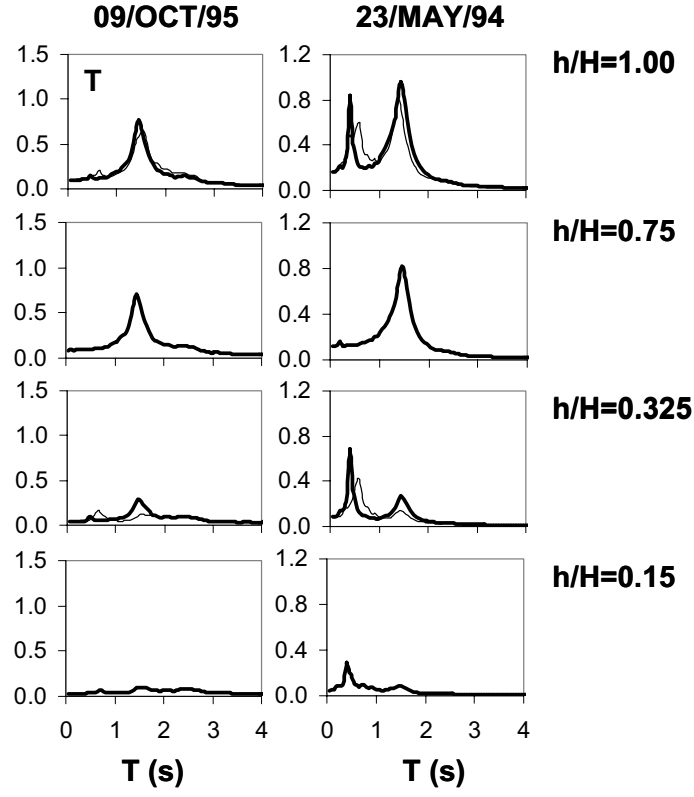

b) Dirección transversal

Figura 13. Respuesta en diferentes niveles del edificio PI (línea delgada, observada y línea gruesa, calculada) ubicado en terreno firme ante los sismos del 09/Oct/95 y 23/May/94

Las amplificaciones en los pisos superiores con respecto al suelo varían entre dos y seis veces, siendo mayores las amplificaciones en estructuras con $\mathrm{T}<1 \mathrm{~s}$ ante sismos de falla normal y para estructuras con $\mathrm{T}>1 \mathrm{~s}$ debido a sismos de subducción. Igualmente, es importante considerar, el tipo de estructuración del edificio: ante sismos de falla normal para estructuras con periodos mayores a un segundo las amplificaciones en la azotea son mayores para estructuras que se deforman como vigas a flexión (estructuras con muros o contravientos) con respecto a estructuras que se deforman como vigas de cortante (marcos estructurales).

El riesgo sísmico de estructuras en sitios del valle de México está prácticamente dominado por los eventos de subducción, sin embargo, de acuerdo con lo mostrado en este trabajo, puede esperarse que los sismos de falla normal causen daños en estructuras con periodos estructurales $\mathrm{T}<1.0 \mathrm{~s}$ en terreno firme y en zona de lago en periodos alrededor del segundo modo fundamental del suelo. También se pueden esperar mayores distorsiones en pisos superiores y daños en contenidos de edificios en los diferentes niveles del edificio debido a este tipo de sismos. Para fines prácticos, es muy importante utilizar acelerogramas de diseño de ambas fuentes sísmicas en la evaluación de la respuesta estructural debido a que no sólo se debe contemplar el uso de grandes sismos de subducción sino también considerar los efectos que tienen los sismos de falla normal en la respuesta estructural. 


\section{AGRADECIMIENTOS}

El primer autor reconoce y agradece la beca otorgada por el CONACYT.

\section{REFERENCIAS}

Arredondo, C, E Reinoso y E Miranda, (2004). "Demandas aproximadas para edificios instrumentados en el Valle de México", XIV Congreso Nacional de Ingeniería Estructural, Acapulco, Gro.

Furumura, R y S K Singh, (2002), "Regional wave propagation from Mexican Subduction Zone Earthquakes: The attenuation functions for interpolate and inslab events", Bull. Seism. Soc. Am., 92, No. 6, 2110-2125.

Iglesias, A, S K Singh, J F Pacheco y M Ordaz, (2002), "A source and wave propagation study of the Copalillo, Mexico earthquake of July, $2000(\mathrm{Mw}=5.9)$ : Implications for seismic hazard in Mexico City from inslab earthquakes", Bull. Seism. Soc. Am., 92, No. 3, 1060-1071.

Ishikawa, Y y H Kameda, (1988), "Hazard-Consistent magnitude and distance for extended seismic risk analysis", Proc. of Ninth World Conference on Earthquake Engineering, II, 89-94.

Jaimes, M., M Ordaz, E Reinoso y E Flores, (2004), "Simulación sísmica combinando funciones de Green y análisis probabilístico para una intensidad y distancia incierta de la fuente", III Informe de doctorado en el Posgrado de Ingeniería.

Mahin, S. A. (1980). "Effects of duration and aftershocks on inelastic design earthquake", Proc. Eight World Conference on Earthquake Engineering, San Francisco, Cal, 881-888.

Miranda, E y C J Reyes, (2002), "Approximate lateral drift demands in multi-story buildings with non-uniform stiffness”, Journal of Structural Engineering, Vol. 128, No. 7, pp. 840-849.

Miranda, E y S Taghavi, (2005), "Approximate floor acceleration demands in multi-story buildings. I: Formulation", Journal of Structural Engineering, ASCE, 131, No.2, 203-211.

Nishenko, S P y S K Singh, (1987), “The Acapulco-Ometepec, México earthquake of 1907-1982: Evidence for a variable recurrence history”, Bull. Seism. Soc. Am., 77, 1359-1367.

Ordaz, M, J Arboleda y S K Singh, (1995), "A Scheme of Random Summation of an Empirical Green's Function to Estimate Ground Motions from Future Large Earthquakes", Bull. Seism. Soc. Am., 85, No. 6, 1635-1647.

Ordaz, M, E Miranda y J Áviles, (2000), “Propuesta de espectros de diseño para el DF”, Sociedad Mexicana de Ingeniería Estructural.

Pavón, R V y N C Rodríguez, (1990), "Instrumentación sísmica y uso de elementos disipadores de energía en el comportamiento estructural", Seminario Internacional, Sociedad Mexicana de Ingeniería Sísmica.

Reinoso, E y E Miranda, (2005), "Estimation of floor acceleration demands in high rise buildings during earthquakes", Structural Design of Tall and Special Buildings, 4, No. 2, 107-130.

Singh, S K (2003), "Advances in seismology with impact on earthquake engineering", XIV Congreso Nacional de Ingeniería Sísmica, Conferencia Magistral, León, Gto. 
Taghavi, S y E Miranda, (2005), "Approximate floor acceleration demands in multi-story buildings. II: Applications", Journal of Structural Engineering, ASCE, 131, No. 2, 212-220.

Terán-Gilmore, A (1996), "Performance-Based Earthquake-Resistant Design of Framed Buildings Using Energy Concepts", Tesis de Doctorado, Universidad de California, Berkeley, Cal. 